\title{
Comparison and Compilation of the Vaccine Approval Process of the United States of America and the European Union: A Road Map for the Emerging and Developing Countries in COVID-19 Crisis
}

\author{
Sumana Dosemane Radhakrishna ${ }^{1}$, Ravi Gundadka Shriram ${ }^{2, *}$, Shenaz Z. Khaleeli ${ }^{3}$, Pankaj Dawar ${ }^{3}$, \\ Megha Chellath Kottayi ${ }^{1}$, Akhilesh Dubey ${ }^{1^{*}}$
}

${ }^{1}$ Department of Pharmaceutical Regulatory Affairs, NGSM Institute of Pharmaceutical Sciences, NITTE (Deemed to be University), Mangaluru-575018, Karnataka, INDIA.

${ }^{2}$ Formulation and Development, R\&D Center, Mylan Laboratories Ltd, Bengaluru, Karnataka, INDIA.

${ }^{3}$ PharmaLeaf India Pvt. Ltd., Bengaluru, Karnataka, INDIA.

\begin{abstract}
Background: Vaccines are the-omit complex biological preparations that are meant to be used as a treatment or preventive measures against any specific disease condition. Vaccine research and development is a tedious, time and cost consuming process because of stringent quality assessment procedures. Every year approximately 80-85 $\%$ of the world's children receive vaccines; however, regardless of this success rate each year, approximately more than 3 million die from vaccine-preventable diseases in most of the-omit developing countries. Today the entire world is facing a pandemic COVID-19 and the researchers are putting their best effort to bring a vaccine into the market. Developing a vaccine is a tedious process and emerging vaccine into the market requires a whole lot of process. Purpose: The current study aimed to evaluate the vaccine approval processes of two international pharmaceutical regulatory giants and develop a road map for the progress of vaccines in emerging countries to face the challenges posed by COVID-19. Methods: This article expresses the brief updated report of the regulatory approval pathways involved in the vaccines registration process in the United States of America (USA) the European Union (EU). The comparison study serves to understand the regulatory issues involved during the life-cycle of the vaccine approval process in the USA and the EU. Authors advocate the necessity of harmonised guidelines for the vaccine approval process in developing countries. Conclusion: Authors insist on broad global partnerships between the developed and emerging countries for the supply of affordable vaccines across the globe to face the pandemic called COVID- 19.
\end{abstract}

Key words: Vaccines, Marketing authorization application, USA, EU, COVID-19.

\section{INTRODUCTION}

The novel coronavirus disease (COVID19) is an infectious disease caused by newly discovered coronavirus SARS-CoV-2 rattled the whole world which was originated in China in mid-December 2019. This virus has infected most of the world's population and causing respiratory illness. Currently, several pharmaceutical companies and research organizations worldwide are engaged in preclinical and clinical research for developing effective drugs and vaccines against COVID-19. ${ }^{1}$

Vaccines are the substances which stimulate the body immunity against any particular disease or provide immunity to the body. We can obtain vaccines from different sources like bacteria, virus and microorganisms. Since vaccines are administrated directly to the body, they must undergo a rigorous review in the laboratory to ensure that the vaccine is safe and effective against the
Submission Date: 15-10-2020; Revision Date: 04-12-2020; Accepted Date: 26-05-2021

DOI: 10.5530/ijper.55.2s.106 Correspondence: Dr. Akhilesh Dubey Department of Pharmaceutical Regulatory Affairs, Formulation and Development, NGSM Institute of Pharmaceutical Sciences, NITTE (Deemed to be University),

Mangaluru-575018

Karnataka, INDIA

Phone: +91-9986893787

E-mail: akhilesh@nitte.edu.in

Dr. Ravi G.S,

R\&D Center,

Mylan Laboratories Ltd.

Bengaluru-560105,

Karnataka, INDIA.

Phone: +91-9535282990

E-mail: ravigs1991@gmail.

com

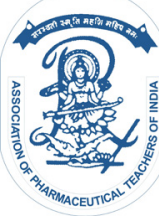

www.ijper.org 
particular disease condition. Vaccines are the complex molecules because of the biological nature of their sources; hence they must undergo extensive testing for the characterisation. Vaccine regulations are stringent because of their complexities and their effects on the patients. We must further review vaccines for the potential side effects after the administration. ${ }^{1}$ The regulatory requirements of vaccines are still region or countryspecific and day by day, these regulations becoming more complex. Therefore, there is a need to develop harmonized guidelines for the vaccine approval process in developing and emerging countries. Center for Biologics Evaluation and Research (CBER) of the United States Food and Drug Administration (USFDA) deals with the vaccines and related products under section 351 of the Public Health Service Act. Biologics License Application (BLA) in FDA Form 356h is used to apply to obtain approval to manufacture and market the vaccines in the USA. ${ }^{2-6}$ European Union (EU) follows a Marketing Authorisation Application (MAA) procedure to regulate the approval process of the vaccines. ${ }^{7}$

\section{Generalised vaccine approval process}

The general stages of the development cycle of a vaccine can be divided into the exploratory stage, pre-clinical stage, clinical development and regulatory review and approval stages. ${ }^{9}$ The general stages of the development cycle of a vaccine are summarised in the Figure 1.

\section{Exploratory stage}

The initial stage in the development of the vaccine is known as an exploratory stage where extensive research will be taking place on a specific type of disease and its conditions in order to find out and identify the certain immunogen or antigen which will prevent the disease or cure the disease. The exploratory period lasts up to two to four years.

\section{Pre-clinical stage}

A pre-clinical stage starts with the development of manufacturing units for vaccines. The manufactured vaccines are then subjected to non-clinical studies. Non-clinical studies are done through in-vitro studies, in-vivo studies method along with the animal testing to understand the results from toxicity testing and pharmacological effects. After the non-clinical studies, the manufacturers must apply for Investigational New Drug (IND) application for testing the vaccine product on human subjects. The pre-clinical stage extends up to one to two years.

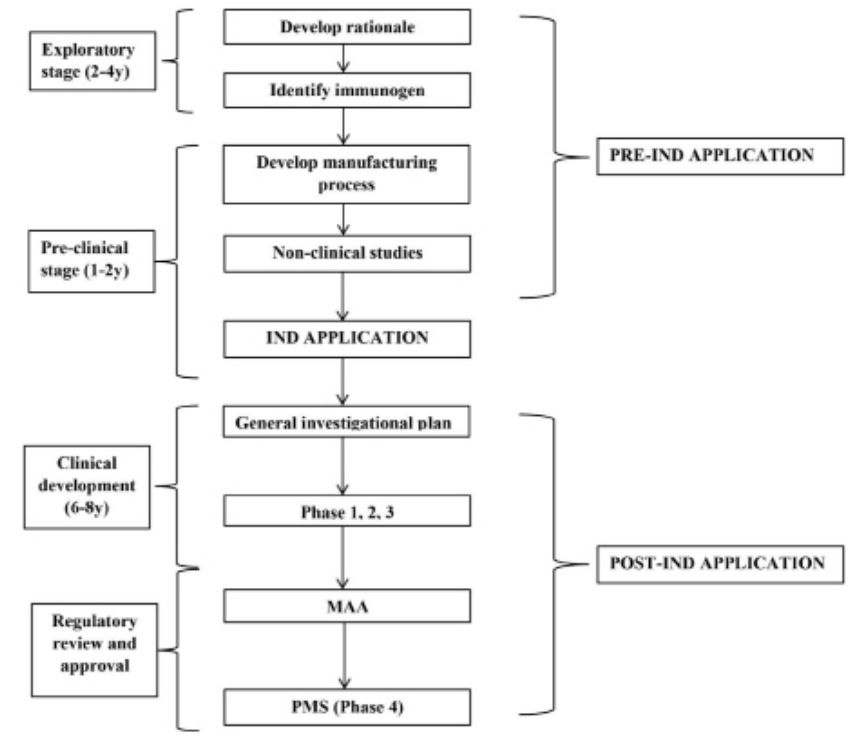

Figure 1: The general stages of the development cycle of a vaccine.

\section{Clinical development}

Clinical development stage begins with the approval of IND. A general investigation plan must be done in order to conduct the clinical trials of vaccines in human subjects. The different phases of the clinical trials like Phase 1, Phase 2 and Phase 3 must be conducted after the approval from the regulatory authority. This phase of the clinical development will take up to six to eight years.

\section{Regulatory review and approval}

Regulatory review and approval stage begins after the Phase 1, Phase 2 and Phase 3 clinical trials. Post marketing surveillance will be conducted at this stage after obtaining market authorization application for vaccines before being sold. However, this part is discussed in detail in later sections of the article.

\section{Vaccine Regulation in the USA}

Vaccine and related products are regulated by the CBER under section 351 of the Public Health Service Act. The registration process of the vaccine is done by applying BLA in FDA Form 356h. ${ }^{10-12}$

\section{Regulatory review and approval}

After the Phase 3 trials, the manufacturer must file for approval for manufacture and market the vaccine in a broad population by applying for CBER of USFDA in FDA Form 356h, which is biologics license application. This biologics license application takes up to 6 to 12 months, depending on the review method, which 
is a priority and standard review. After the approval process, phase 4 or post-marketing surveillance will begin. If any adverse event or effect is observed after the administration of the vaccine, it must be reported to the Vaccine Adverse Event Reporting System (VAERS) within 15 days.

\section{Biologic License Application Process (BLA)}

The biological license application is to market the manufactured vaccine product in the US. This application is submitted in the FDA Form $356 \mathrm{~h}$ of section 351 of the Public Health Service Act. The process of application is initiated on day 1 by taking a decision to file BLA. Meetings must be planned on the $45^{\text {th }}$ day during the application submission process. A time period of 60 days will be provided to the manufacturer to file the BLA with the necessary required dossier documents and to schedule the inspection time period. FDA will determine whether an applicant qualifies priority or standard review. The manufacturer can also request priority review if the vaccine product provides significant improvements to public health. Priority review will take up to 6 months to review the vaccine product, whereas the standard review takes up to 12 months to review the vaccine product. FDA will also inform the applicant within day 60 if it is designated with priority review. In case of a standard review, it will be informed within day 74. If the FDA finds any potential issue in the vaccine product, it will notify the manufacturer on Day 75. The manufacturer should involve in mid cycle meeting along with the Vaccines and Related Biological Products Advisory Committee (VRBPAC) in order to minimise the risk involved in the vaccine product and report the necessary corrective action to FDA. The inspection will be done during this review period and the complete review will be documented. Complete action will be taken for compliance checking of the vaccine product. The application will be circulated for the approval and approved. This whole process is briefly summarised in Figure 2.

\section{ADR reporting of vaccines}

Adverse drug reaction (ADR) reporting of vaccines is done through VAERS form, which is used to report any adverse experiences associated with the use of vaccine products. VAERS is a national vaccine safety surveillance program co-sponsored by the FDA and the Centers for Disease Control and Prevention (CDC). Vaccine adverse event reporting is done through online submission or fax or by mail. Reporting must be done within 15 days of identifying the adverse event.

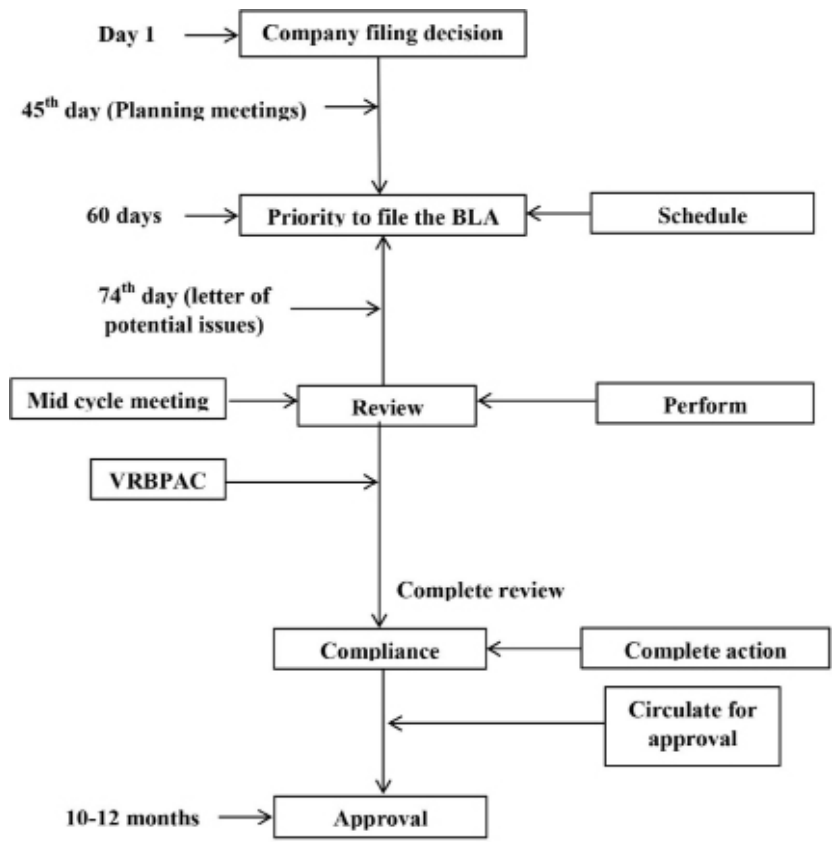

Figure 2: Biological License Application (BLA) flow chart.

\section{Vaccine regulations due to COVID-19 crisis in the USA}

In the present scenario, the FDA has taken vital steps to ensure the prompt development of safe and effective vaccines to avert COVID-19 by guiding with recommendations for those developing COVID-19 vaccines. The FDA issues new guidance documents for developing safe and effective COVID-19 vaccine by providing considerations regarding Emergency Use Authorization (EUA) of an investigational vaccine. The Secretary of Health and Human Services must make a declaration of emergency or threatjustifying authorisation of emergency use before the FDA can issue a EUA for any specific product. On $4^{\text {th }}$ February 2020, the Secretary made this determination concerning COVID-19, the vaccines licensed in the United States should meet the statutory and regulatory requirements for vaccine development and approval, quality, manufacture and control (section 351(a) of the Public Health Service Act (PHS Act), (42 U.S.C. 262)). The vaccine product must be adequately characterized and its manufacture in compliance with applicable standards including current good manufacturing practice (cGMP) (section 501(a) (2)(B) of the FD and C Act (21 U.S.C. 351(a)(2)(B)) and 21 CFR Parts 210, 211 and 610). Understanding of SARS-CoV-2 immunology and specifically vaccine immune responses that might predict protection against COVID-19, is currently limited and evolving. Hence, the evaluation of immunogenicity is a crucial component of COVID-19 vaccine development. 
The ideal and compatible clinical trial designs might accelerate the clinical development programs for COVID-19 that allow for selection between vaccine candidates and dosing regimens and more rapid progression through the usual phases of clinical development. ${ }^{13-15}$

\section{Stage 1 Research and Development}

Usually, the time taken for developing a vaccine is 2 to 4 years, but in the case of coronavirus, it has been very quick since the Chinese government had shared the genetic sequence of the virus.

\section{Stage 2 Pre-Clinical}

After the first stage, the vaccine is tested on animals and plants to evaluate their efficacy and functioning and their immune responses has been analysed.

\section{Stage 3 Clinical}

This stage is the most significant and vital stage in the development of the vaccine as its efficacy is tested on humans. This consists of 3 phases. In Phase 1, vaccine is administered to a small group of people and tested to check whether antibodies have developed inside their bodies or not. In Phase 2 the vaccine is administered on hundreds of people and it may take 6-8 months. The candidates are analysed on developing an immune response against the disease. In Phase 3 vaccine is conducted on thousands of people and can take 6-8 months.

In order to begin clinical trials, the vaccine sponsor should obtain an approved investigational new drug application (IND). However, therapeutic and vaccine developers should seriously consider asking the FDA for a pre-IND meeting. They may reduce time to market by identifying unnecessary studies and ensuring that studies are designed to provide useful information and can gain the agency's support for a particular development strategy.

\section{VACCINE REGULATION IN THE EU}

The vaccine regulation process in the $\mathrm{EU}$ to assess the quality, efficacy and safety consist of marketing authorization, registration, quality assessment and pharmacovigilance and risk assessment plan. ${ }^{16,17}$

\section{Marketing authorization}

Marketing authorisation is granted by the Committee for Medicinal Products for Human Use (CHMP) after reviewing the entire dossier for the quality, safety, efficacy and the risk-benefit involved in the product.

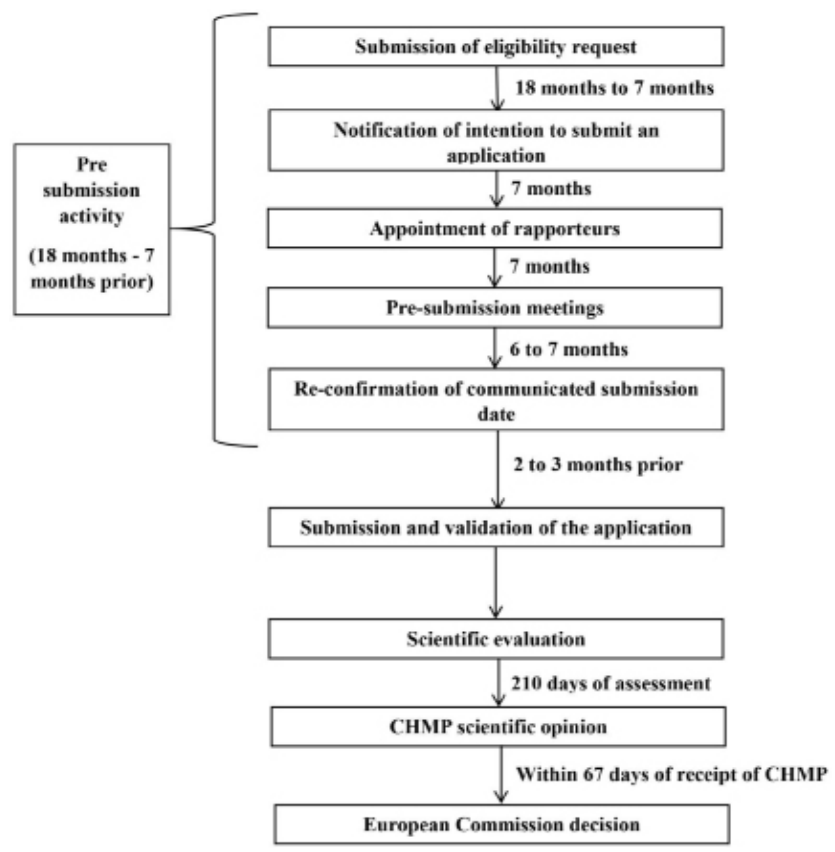

Figure 3: Marketing Authorisation Application (MAA) of EU.

Review of the dossier for the compliance in the areas like manufacturing, clinical and laboratory testing should be prior approved by the regulatory authority. The MAA approval process is briefly summarized in Figure 3.

\section{Registration}

Vaccine approval processes in the EU are done through centralised, decentralised, mutual recognition and nationalised procedures. The manufacturer generally prefers the centralised procedure while applying for the marketing authorisation application for the vaccine product. Since centralised procedure is the single marketing authorization approval of the vaccine product throughout the EU which allows the vaccines readily available to the patients and the healthcare system. CHMP will evaluate the single application which is sent to the European Medical Agency (EMA) for marketing vaccine product in entire EU.

\section{Quality assessment}

After obtaining marketing authorisation, vaccines will be assessed for quality before release into the market. Manufactures, an official European control laboratory, European Pharmacopoeia Secretariat within the European Directorate for Quality of Medicines (EDQM) facilitates the quality assessment process.

\section{Pharmacovigilance and risk management plan}

Vaccines are monitored after release onto the market for adverse events and ensure the safety profile established 


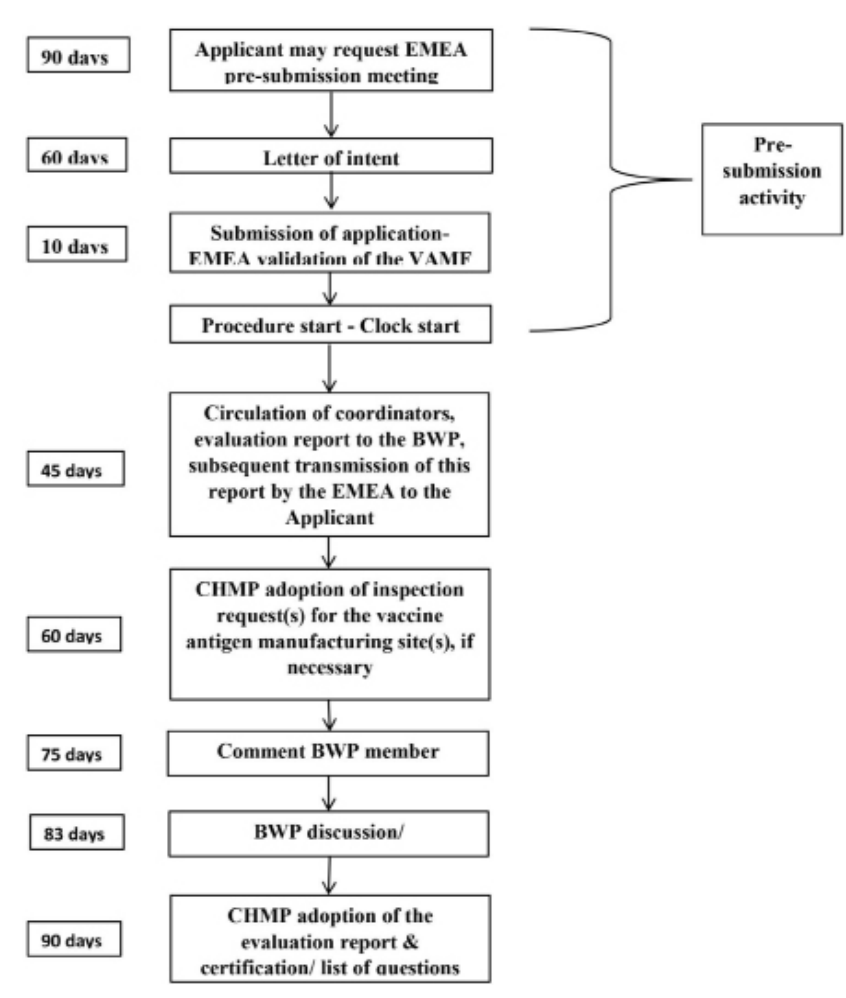

Figure 4: Vaccine Antigen Master File (VAMF) approval process.

during the product development. The risk management plan also set up to detect rare events that may not have been apparent during the clinical development.

\section{Vaccine Antigen Master File (VAMF)}

In the case of the centralised procedure, the vaccine manufacturer must apply for the Vaccine Antigen Master File (VAMF) certification prior to the MAA approval (European Medicines Agency, 2005). The VAMF approval process is briefly summarized in Figure 4. Pre-submission meeting with the scientific committees will enable the manufacturers to opt for the marketing authorisation procedure and also VAMF submission process. Generally centralised procedure is preferred and the scientific evaluation of the submitted dossier will be done by the CHMP (EMEA, 2005). Vaccine adverse event is reported through the Eudra Vigilance system in the EU. The adverse event can be reported by the manufacturer or the patient or the physician. The report will be assessed under the Eudra Vigilance system and CHMP will act on the report to reject or the approval of the product from the market (European Medicines Agency, 2003). Table 1 helps to understand the differences and the similarities observed in the marketing authorization requirements for vaccines in the USA and EU.

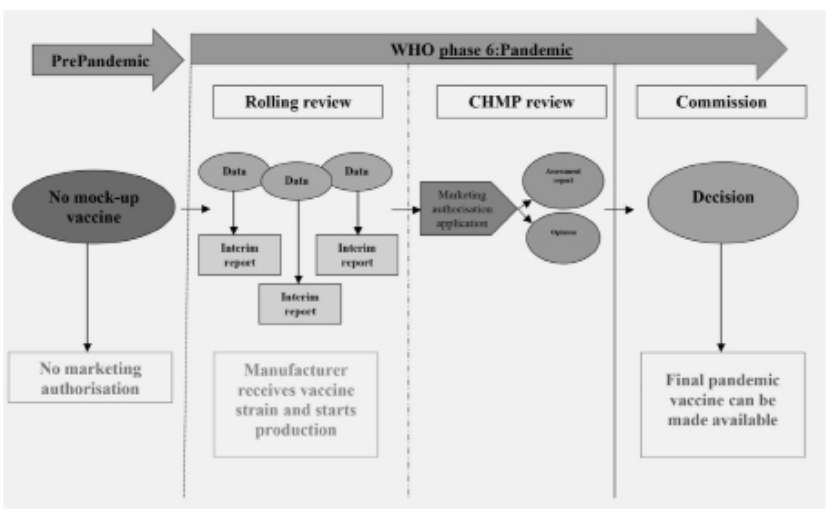

Figure 5: Authorisation of pandemic flu vaccines using the 'emergency' procedure by European Medicines Agency?

\section{Vaccine regulations due to COVID-19 crisis in the EU}

The European Medicines Agency authorizes the vaccine promptly compare to the previous regulations in which 18 to 24 months usually required for the authorisation of medicine in the EU. ${ }^{18}$ The two main procedures for the approval of pandemic influenza vaccines are:

- Mock up procedure: This method allows a vaccine to be developed and approved before a pandemic, based on the information produced with a virus strain.

- Emergency procedure: This method allows fast track approval of a vaccine after the pandemic has occurred (Figure 5). The authorisation for these vaccines takes less time than routine vaccines. The information submitted by the manufacturer is assessed in an accelerated time frame. The regulation is suggesting that the companies developing new vaccines need to procure more data that are needed to change the virus strain in a mock-up vaccine. In this procedure, companies will adopt the rolling review process, providing data on vaccines under development as they become available rather than waiting until they have collected the dossier of data.

The vaccine developers carry out the tests in three phases of clinical trials, considering a significant number of people in each phase. The regulators set the procedures and protocols for the programme. The medicines regulatory authority can inspect the premises and verify the information that the vaccine developer provides. In addition to this they can carry out tests to ensure the batches of vaccines are of the expected quality before release onto the market. As per the acceptance criteria pre-defined by the authorities, the companies are required to conduct stringent testing before release onto the market. 
Table 1: Comparison of marketing authorization application in USA and EU markets for vaccines.

\begin{tabular}{|c|c|c|}
\hline & USA & EU \\
\hline Regulatory Agency & Federal Food \& Drug Administration (USFDA) & European Medicine Agency (EMA) \\
\hline Regulating Ministry & Department of Health \& Human Services & European Commission (EC) \\
\hline Regulation & Public Health Service (PHS) Act & European Directives \\
\hline Type of Submission & Online Submission- e-Submission Gateway & $\begin{array}{l}\text { Online Submission- e-Submission Gateway or } \\
\text { Web Client }\end{array}$ \\
\hline Format of Submission & Country Specific & ICH eCTD Format \\
\hline Guidelines & $\begin{array}{ll}> & 21 \text { CFR } 600 \text { - Biological Products: General } \\
>\quad 21 \text { CFR } 601-\text { Licensing } \\
>\quad 21 \text { CFR } 610 \text { - General Biological Products } \\
\text { Standards } \\
>\quad 21 \text { CFR } 610.60 \text { - Labeling Standards } \\
>\quad \text { Guidance for Industry - Content and format } \\
\text { of chemistry, manufacturing and controls } \\
\text { information\& establishment description } \\
\text { information for vaccine/ related product }\end{array}$ & 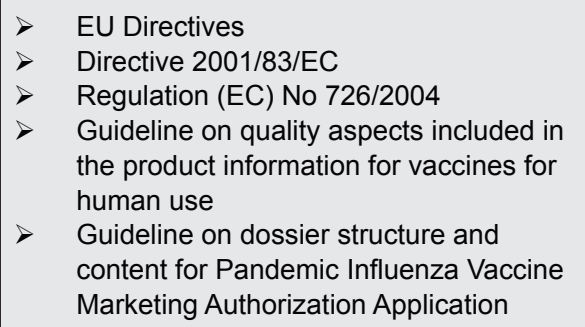 \\
\hline $\begin{array}{l}\text { Classification of Vaccine as per } \\
\text { Source }\end{array}$ & $\begin{array}{ll}> & \text { Live Attenuated } \\
> & \text { Inactivated } \\
> & \text { Subunit } \\
> & \text { Toxoid } \\
> & \text { Conjugate } \\
> & \text { DNA Recombinant Vector }\end{array}$ & $\begin{array}{ll}> & \text { Live Attenuated } \\
> & \text { Killed Inactivated Sub-unit }\end{array}$ \\
\hline $\begin{array}{l}\text { Input Application Form/ } \\
\text { Regulatory Pathway }\end{array}$ & FDA Form 356h & Marketing Authorization Application \\
\hline Output License & New Drug approval & Marketing Authorization Certificate \\
\hline MAA Fees & $\$ 4,154,664$ & $€ 286,900$ \\
\hline Mode of Payment & $\begin{array}{l}\text { Online payment via user fee system } \\
\text { PDUFA user fee cover sheet }\end{array}$ & $\begin{array}{l}\text { Payment done in euro via transfer to agency } \\
\text { bank account }\end{array}$ \\
\hline TAT for Review Process & 6-12 months & 210 days \\
\hline MAA Validity & Perpetual & 5 years \\
\hline Advisory Committee & $\begin{array}{l}\text { Vaccine Related Biological Product Advisory } \\
\text { Committee (VRBPAC) }\end{array}$ & $\begin{array}{l}>\quad \text { Scientific Advisory Group on Vaccine } \\
(\text { SAG-V) } \\
>\quad \text { Vaccine Working Party }\end{array}$ \\
\hline Regulatory Definition & $\begin{array}{l}\text { Vaccine is an immunogen, administration of which } \\
\text { is intended to stimulate the immune system to } \\
\text { result in the prevention, amelioration or therapy of } \\
\text { any disease or infection. }\end{array}$ & $\begin{array}{l}\text { Vaccine is a biological preparation that } \\
\text { improves immunity to a particular disease. }\end{array}$ \\
\hline Quality Certification & NA & VAMF Certification \\
\hline $\begin{array}{l}\text { Country Specific Documents for } \\
\text { Submission }\end{array}$ & Label & Summary of Product Characteristics \\
\hline Labeling Guidelines & $\begin{array}{l}\text { Pre-approval review of label is important. } \\
\text { Guidance for industry FDA review of vaccine } \\
\text { labeling requirements for warnings, use } \\
\text { instructions, \& precautionary information }\end{array}$ & $\begin{array}{l}\text { Guideline on the readability of the labelling } \\
\text { and package leaflet of medicinal products. } \\
\end{array}$ \\
\hline ADR Reporting & $\begin{array}{l}\text { Vaccine Adverse Event Reporting System } \\
\text { (VAERS) }\end{array}$ & EudraVigilance system \\
\hline Post Marketing Requirements & Product Specific PMS Requirements & Pharmacovigilance \& Risk Management Plan \\
\hline
\end{tabular}




\begin{tabular}{|c|c|c|c|c|}
\hline \multicolumn{5}{|c|}{ Table 2: Current status of the vaccine development } \\
\hline Organization & Vaccine Candidates & Clinical Phase & Vaccine type & Headquarters \\
\hline AstraZeneca/ University of Oxford & 1 & III & Recombinant & UK \\
\hline BioNTech/Pfizer & 4 & II & mRNA & Germany/US \\
\hline Gamaleya Federal Research Center & 2 & II & Recombinant & Russia \\
\hline Immodulon Therapeutics & 1 & II & Inactivated Virus & UK \\
\hline Imperial College London & 1 & 1 & mRNA & mRNA \\
\hline Curevac & 1 & 1 & Germany \\
\hline
\end{tabular}

In the global market, European vaccine developers are considered among the most advanced. In the recent development a vaccine developed by AstraZeneca and the University of Oxford began phase 3 trials in Brazil (Table 2). However, most vaccine developers are focusing on a single vaccine candidate, the Gamalaya Federal Research Center and BioNTech seem to be trying with more than one. BioNTech, for example, is testing four messenger RNA (mRNA) vaccine candidates in parallel. ${ }^{18-20}$

\section{A ROAD MAP FOR THE DEVELOPMENT OF VACCINES IN EMERGING COUNTRIES}

There are primarily two types of market existing in this sector, developed or mature and emerging or developing market. Each sector has its structures and dynamics. The countries like Brazil, Russia, India, China and South Africa (BRICS) dominate the markets at present in the pharmaceutical sector. In these countries, we found exports of pharmaceutical products to be low than developed countries in the past decade; however, the growth rate is faster than in developed countries. Emerging manufacturers are playing an active role such as in India, Panacea Biotech, Shantha Biotechnics, Bharat Biotech, Biological E Ltd., Serum Institute of India; In China, Chengdu, Shanghai (SIBP) Sinovac, Shenzhen AVP, Shenzhen Kangtai; In South Korea, Berna Green Cross (Berna), LG Life Sciences; In Brazil, Bio-Manguinhos and Butantan Institute; In Mexico and Cuba, Birmex and CIGB, Instituto Finlay respectively. The following suggestions can help to develop the vaccines in emerging countries. ${ }^{21}$

\section{People-focused}

Ensuring the future vaccine system deliver sustained and high-quality services to an international health system. Placing people and the families as the priority for the design, management and delivery of immunization.

\section{Country centred}

Ensuring the availability and access to the vaccination and immunization to all the geographical locations without any discrimination. Each country is responsible for developing and supporting the immunization initiative essential to protect the health of its population.

\section{Broad partnership}

Global, regional alliances will maximize the impact on sustainable development and contribution to support the immunization agendas or activities. Partnerships also help in the global supply and transition of affordable vaccines through different supporting programmes, e.g.: Gavi/ polio.

\section{Data-driven}

Encouragement for the novel vaccine technologies and sharing the knowledge will help to practice the evidence-based or the cost-effective use of the resources used during the research and development of the vaccination. Evidence-based practice will help to understand the data and implementation of the research for any disease threats.

\section{AUTHORS PERSPECTIVE}

Theopinionsaregivenby theexpertsof thepharmaceutical industry and the deliberations of the authors working on the project. The authors are promoting the logical solution to harmonise the registration procedure to ensure the reachability of the vaccines to the developing countries. The perspectives are:

- The authors believe that developing a vaccine in the current situation is mandatory; however, maintaining a standard of procedures and quality assessment on testing is equally important. Different countries are in urge to develop a vaccine to tackle this deadly infectious disease and countries like the USA and EU have set forward new guidance documents for manufacturing such vaccines and 
approving them. Currently, Indian researchers are put on pressure to develop the vaccine, but the quality and safety of the vaccine is an essential factor to be considered before releasing it to the general population. The biggest challenge before the vaccine developers is to accelerate and harmonize processes to ensure the products are safe and effective; before reaching billions of people in the world. Past experiences, such as H1N1 outbreak in 2009, shows that when drugs were available but not able to reach everyone equally. Seventy-five countries have submitted expressions of interest to safeguard the health of their people and with other nations through joining the COVAX Facility. Itis a mechanism designed to guarantee rapid, fair and equitable access to COVID-19 vaccines worldwide. This mechanism works by sharing the risks associated with vaccine development considering the investment in manufacturing. Thus, vaccines can be deployed at a large scale as soon as they are proven successful, to end the deadly pandemic by 2021 .

- Vaccines that are developed with the novel therapeutic approaches in recent times are poised to counter the non-infectious chronic diseases. Therefore, there is a desire to understand the global perspective of the regulated and emerging market of vaccines. The regulated markets specify certain standards to demonstrate the products to be safe and effective for human use by the statutory requirements during the approval process. Regulated countries follow the ICH guidelines for the stability programme and the climatic zones. Emerging markets are developing the best standard regulation to demonstrate a safe and effective drug product. Regulatory requirements in the case of the stability programme are difficult since some emerging countries fall in different temperature zones. The authors feel that the pre-submission meetings and the scientific advice from the experts are imperative for the regulated markets prior to the submission of the technical dossiers.

- The authors believe that the proper alignment is recommended in the CTD numbering system, which serves to streamline faster access to vaccines in developing countries. The authors indicate that it requires the standardised evaluation procedure and a standard template for application forms for the smooth functioning that brings to better convergence of file content. The authors realised that it requires the regulatory giants such as USFDA and EU to share the information with the immunization community such as regulators, programme managers, country-wise regulatory authorities, donors and other stakeholders. Let us discuss the case of India, where India is emerging as the vaccine hub of the world and it has a key role in the vaccine market. Indian market focuses on global product development by understanding the varying regulatory requirements globally and observing the harmonised ICH guidelines during the development of the product. The regulatory pathway for vaccines in India is currently laden with delays since all vaccines are categorised under a new drug classification even if they are the follow-on products to currently approved vaccines. Let us follow the statistics where in India, approximately 0.5-1 million lives are lost every year because of vaccine-preventable deaths. These figures are terrible and threefold to those in the USA, Germany, China and Brazil. Statistics also reveal that India accounts for less than $2 \%$ of the global market for vaccines and per capita spending on vaccines is extremely low compared to the USA and many European countries. Therefore, the authors advocated the harmonisation of the vaccine approval process.

- The authors emphasizes the labelling and packaging requirements of different countries, especially for the emerging and developing markets vary according to the specific country. Guidance document must be explicitly introduced specifically to the region to overcome the issues related to harmonization of the labelling. Towards this step, the Developing Countries Vaccine Manufacturers Network (DCVMN) and the International Federation of Pharmaceutical Manufacturers and Associations (IFPMA) and a group of a regulatory expert from industry should work on the issues of labelling and packaging requirements. The authors suggest a standard template for labelling vaccines across the globe with a single, uniform label format instead of a four label format.

- The authors believe that the fundamental part of the vaccine life cycle process is quality. It encourages manufacturers to figure out the appropriate study data and analytical designs for the suitable vaccine product by the corresponding regulatory agency. Harmonized guidelines must be adhered to understand the global regulatory requirementin terms of handling; storage and distribution of the temperature-sensitive product are not compromised. Good distribution and storage practices and supply chainmustbeencouraged by the regulatoryauthorities to oversight and maintain the documentation of the quality procedures. Risk assessment and risk 
management, benefits decision must be adopted based on the scientific evidence to control and prioritise to make decisions to prevent the unwanted effects caused by the vaccines.

- The authors suggest that healthy communication is required to develop between the regulators and sponsors that may lead to an increased rate of registration and market access. The combination of emerging and established regulators should be established such as ANVISA (Brazilian regulatory authority), CDSCO (Indian regulatory authority), MCC (South African regulatory authority), EMA (European Medicine Agency) and USFDA (U.S regulatory authority) to meet regularly to share the information regarding the vaccine approval process.

- The authors believe that the key areas for improvementintheareabeingdiscussedaretopromote the development of new vaccines with easier use of technologies to meet public health priorities and also ensuring the vaccines and delivery technologies with assured quality. The price fixation is an important issue that has to be resolved in order to bring better transparency. The authors find that the disparity exists between stable and unstable countries and that may continue to remain due to their political and internal problems. Perhaps this is the major shortcoming that prevents advance research in such regions. Nevertheless, stable countries should continue support to maintain reliable immunization services in developing and fragile countries.

- The authors believe that the manufacturers should set the agenda for the development of new and improved vaccines with a proper delivery system. The agenda of manufacturing should address public health priorities of developing countries. Further research holds on to develop novel vaccines enabled with easier-to-use immunization technology and collaboration with expert groups around the world. Authors emphasis on to establish standards and global norms for vaccines to safeguard the quality, safety and efficacy of vaccines. In addition to this easier-to-use immunization technology with the streamline regulatory processes for the registration in developing and emerging countries. The authors believe that the developed world should support emerging countries to establish wellequipped pharmacovigilance system for adverse event reporting to improve vaccine safety.

- From the last decade, global immunization coverage has increased and currently cervical cancer, diphtheria, hepatitis B, measles, polio, haemophilus influenza type $\mathrm{B}$ infections, rotavirus diarrhea, rubella and tetanus are preventable by vaccines. From the current norm in the last ten years, financial support has been given by the global health community to improve the immunization in developing countries. As financial support plays a pivotal role in immunization improvements in emerging countries. The author believes that global funding is necessary in this regard.

\section{CONCLUSION}

Vaccines are the critical biotechnological innovation that possesses an enormous contribution to global health outcomes. A rigorous regulatory procedure has to be followed to assess quality, safety and efficacy before releasing into the market. By following standardised procedures and regulatory approval of safe and effective vaccines in a harmonized way will improve the health of the individuals in emerging or developing countries. Understanding the regulations of countries like the USA and EU will undoubtedly help emerging countries to develop vaccines in a regulated manner. The vaccine developers and manufacturers have to follow the proper marketing authorisation and access strategy along with the suitable active ingredient to develop a vaccine against COVID-19. The vaccine regulation is stringent because of their complexities as it produces the effects after administering the vaccine to the patients. Hence, the objective of the current discussion was to understand the guidelines and MAA requirements of vaccines in the United States and the European Union. This study helps to bring out harmonised registration procedures amongst the guidelines from the US and EU. We require a harmonised registration procedure to ensure the reachability of the vaccines promptly to the global population.

\section{ACKNOWLEDGEMENT}

The authors wish to acknowledge the NGSM Institute of Pharmaceutical Sciences, NITTE (Deemed to be University), Mangaluru, Karnataka, India for providing necessary facilities to carry out this project. The authors are also thankful to PharmaLeaf India Pvt. Ltd., Bengaluru, Karnataka, India for their technical assistance.

\section{CONFLICT OF INTEREST}

The authors confirm that this article content has no conflict of interest. 


\section{ABBREVIATIONS}

USA: United States of America; EU: European Union; CBER: Center for Biologics Evaluation and Research; BLA: Biologics License Application; MAA: Marketing Authorisation Application; IND: Investigational New Drug; VAERS: Vaccine Adverse Event Reporting System; CHMP: Committee for Medicinal Products for Human Use; VRBPAC: Vaccines and Related Biological Products Advisory Committee; EMA: European Medical Agency; FDA: Food and Drug Administration; CDC: Centers for Disease Control and Prevention; VAMF: Vaccine Antigen Master File; EC: European Commission; PHS: Public Health Service; VRBPAC: Vaccine Related Biological Product Advisory Committee; SAG-V: Scientific Advisory Group on Vaccine; DCVMN: Developing Countries Vaccine Manufacturers Network; IFPMA: The International Federation of Pharmaceutical Manufacturers and Associations; ANVISA: National Sanitary Surveillance Agency; CDSCO: Central Drugs Standard Control Organization; MCC: The Medicines Control Council; SARS-CoV: Severe acute respiratory syndrome-related coronavirus; COVID: Coronavirus disease.

\section{REFERENCES}

1. Pollard VT, Ryan MW, Tschammler D, Grieb J. Fast track for COVID-19 Drug and vaccine approval in the United States and Europe. National Law Review. 2020

2. Coronavirus (COVID-19) update: FDA takes action to help facilitate timely development of safe, effective COVID-19 vaccines [internet]. U.S. Food and Drug Administration; 2020 [cited Aug 5 2020]. Available from: https://www.fda. gov/news-events/press-announcements/coronavirus-covid-19-update-fdatakes-action-help-facilitate-timely-development-safe-effective-covid.

3. U.S. Department of Health and amp; Human Services, Vaccine Basics; 2017. Available from: https://www.vaccines.gov/basics [accessed Aug 18 2020].

4. Philadelphia: College of Physicians of Philadelphia. Vaccine development, testing and regulation. The history of vaccines; 2018. Available from: https:// www.historyofvaccines.org/content/articles/vaccine-development- testing and-regulation [accessed Aug 18 2020].

5. Centers for Disease Control and Prevention. Vaccine testing and the Jan 17 approval process; 2014. Available from: https://www.cdc.gov/vaccines/ basics/test-approve.html [accessed Aug 20 2020].

6. U.S. Food and amp; Drug Administration, vaccines; 2018. Available from: https://www.fda.gov/vaccines-blood-biologics/vaccines [accessed Aug 21 2020].
7. European Medicines Agency. Research and development [cited Aug 19 2020]. Available from: https://www.ema.europa.eu/en/human-regulatory/ research-development.

8. U.S. Food and amp; Drug Administration. Center for Biologics Evaluation and Research (CBER); 2019. Available from: https://www.fda.gov/aboutfda/office-medical-products-and-tobacco/center-biologics-evaluation-andresearch-cber [accessed Aug 21 2020].

9. U.S. Food and amp; Drug Administration, Application to Market a New or Abbreviated New Drug or Biologic for Human Use; 2018. Available from: https://www.fda.gov/media/72649/download [accessed Aug 26 2020].

10. U.S. Food and amp; Drug Administration, Instructions for Filling out Form FDA $356 \mathrm{~h}$ - application to Market a New or Abbreviated New Drug or Biologic for Human Use; 2018. Available from: https://www.fda.gov/media/84223/ download [accessed Aug 28 2020].

11. U.S. Food and amp; Drug Administration, Vaccine Adverse Events; 2018. Available from: https://www.fda.gov/vaccines-blood-biologics/report-problemcenter-biologics-evaluation-research/vaccine-adverse-events [accessed Aug 29 2020].

12. U.S. Department of Health and amp; Human Services, Checklist of information to complete the VAERS form [cited Sep 6 2020]. Available from: https://vaers. hhs.gov/docs/VAERS\%202.0_Checklist.pdf.

13. Fast track for COVID-19 Drug and vaccine approval in the United States and Europe [internet]; 2020. National Law Review. Available from: https://www. natlawreview.com/article/fast-track-covid-19-drug-and-vaccine-approvalunited- states-and-europe [accessed on Sep 5 2020].

14. Coronavirus (COVID-19) update: FDA takes action to help facilitate timely development of safe, effective COVID-19 vaccines [internet]. U.S. Food and Drug Administration; 2020 [cited Sep 5 2020]. Available from: https://www. fda.gov/news-events/press-announcements/coronavirus-covid-19-updatefda-takes-action-help-facilitate-timely-development-safe-effective-covid.

15. Development and licensure of vaccines to prevent COVID-19 [internet] U.S. Food and Drug Administration; 2020 [cited Sep 6 2020]. Available from: https://www.fda.gov/regulatory-information/search-fda-guidance-documents/ development-and-licensure-vaccines-prevent-covid-19.

16. Authorisation procedures. European Medicines Agency [internet]. European MedicinesAgency; 2020 [cited Sep 7 2020]. Available from: https://www.ema. europa.eu/en/authorisation-procedures.

17. Approval of vaccines in the European Union [internet]; 2020. European Vaccination InformationPortal. Available from: https://vaccination-info.eu/en/ vaccine-facts/approval-vaccines european-union [accessed on Sep 8 2020].

18. Smith J. The European fight against the Covid-19 coronavirus [internet] 2020. Labiotech.eu [cited Sep 9 2020]. Available from: https://www.labiotech. eu/medical/coronavirus-vaccine-treatment-diagnostics/.

19. Global leaders unite to ensure everyone everywhere can access new vaccines, tests and treatments for COVID-19 [internet]; 2020. Who.int. everywhere-can-access-new-vaccines-tests-and-treatments-for-covid-19 (accessed on 9 September 2020). Available from: https://www.who.int/newsroom/detail/24-04-2020-global-leaders-unite-to-ensure-everyone-.

20. More than. Who.int. 2020;150 countries engaged in COVID-19 vaccine global access facility [Internet]:than-150-countries-engaged-in-covid-19-vaccineglobal-access-facility.

21. World Health Organization. Developing together the vision and strategy for immunization 2021-2030; 2019. Available from: https://www.who.int/ immunization/ia2030_Draft_Zero.pdf [accessed Sep 12 2020]. 
PICTORIAL ABSTRACT

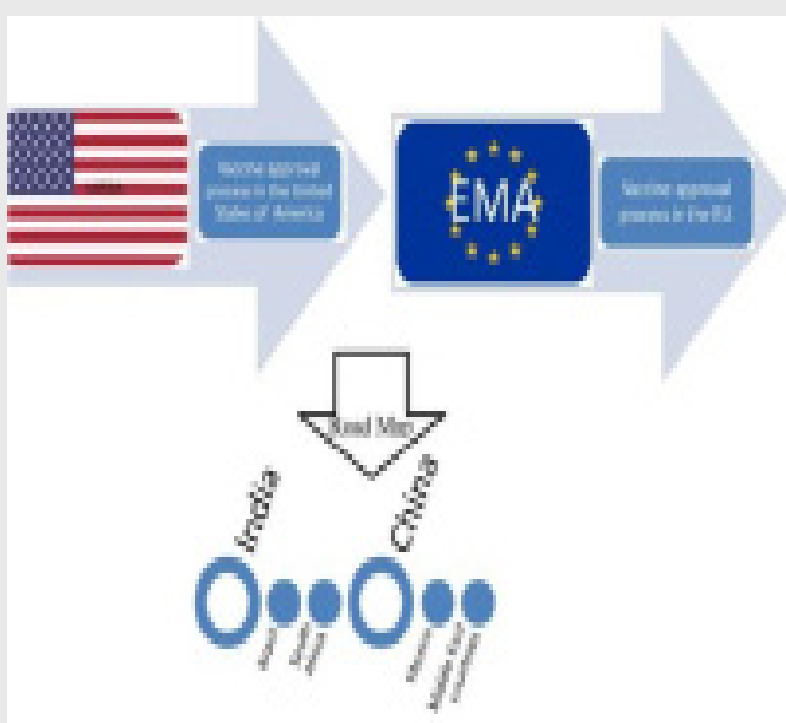

\section{SUMMARY}

The future of the study lies in the area of vaccine development as infectious diseases such as SARS$\mathrm{CoV}$; antimicrobial resistance is promising areas that could be progressed. A higher number of vaccines should be available for the people of emerging and developing countries throughout the human life cycle. Immunization will become an integral part of the disease prevention strategy and delivered more frequently in schools, homes and pharmacies. The promising area that is more likely to develop is easier-touse immunization technology which will enable more comfortable usage of such vaccines by the common man (non-expert health professional). The two most developed regulatory agencies (USFDA and EMA) should come together and ensure the availability of the vaccines for the rest of the world.

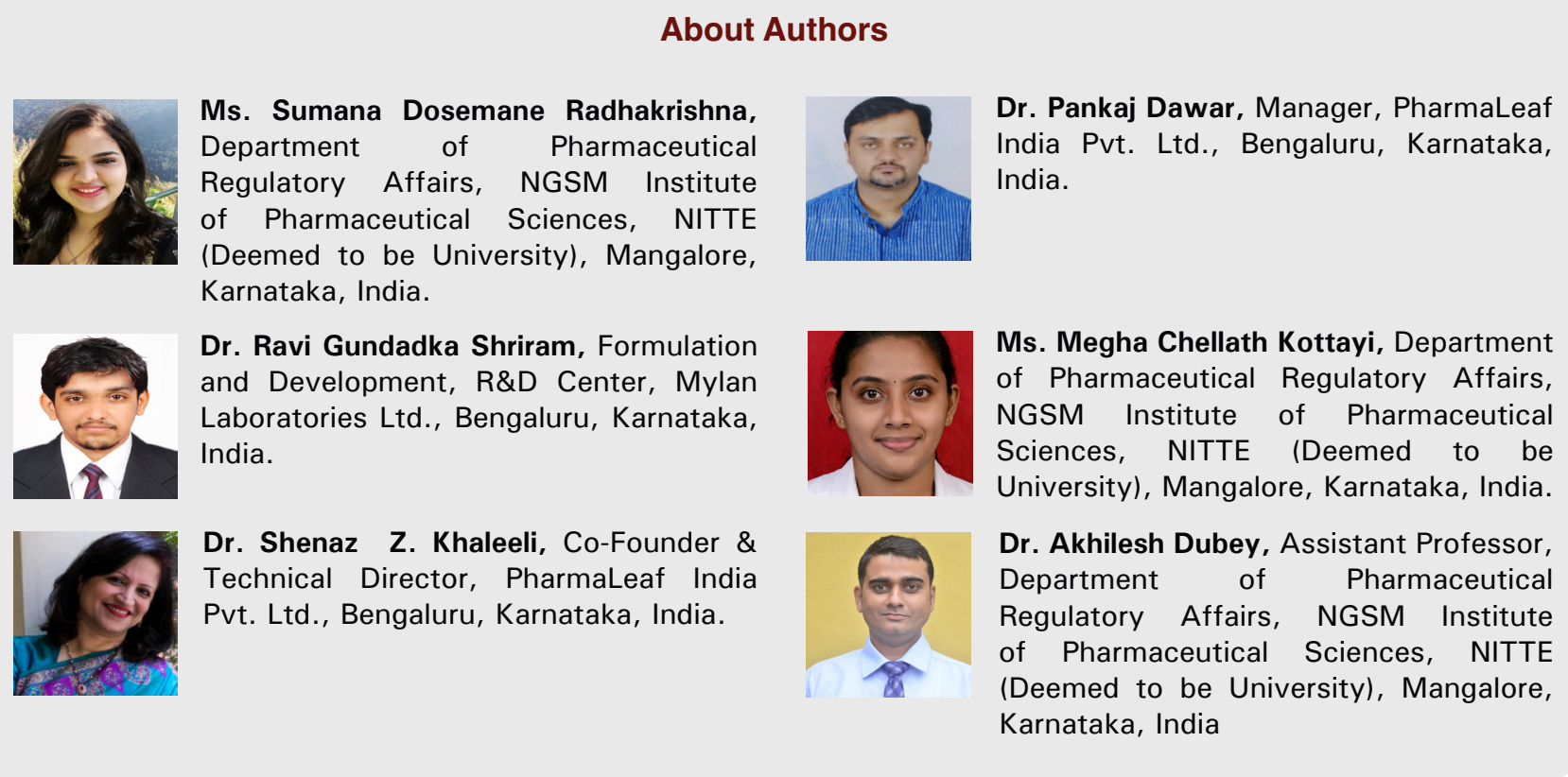

Cite this article: Radhakrishna SD, Shriram RG, Khaleeli S, Dawar P, Kottayi MC, Dubey A. Comparison and Compilation of the Vaccine Approval Process of the United States of America and the European Union: A Road Map for the Emerging and Developing Countries in COVID-19 Crisis. Indian $\mathrm{J}$ of Pharmaceutical Education and Research. 2021;55(2s):s353-s363. 